IC Vol. $5 N^{\circ}$ 2: pp. 267-273, 2014

\title{
“TE DOY MIS OJOS” PERMITE SENSIBILIZAR DERECHO A LA VIDA EN LA VIOLENCIA FAMILIAR*
}

\author{
"TAKE MY EYES" ALLOWS RAISES THE LAW TO LIFE \\ ON FAMILY VIOLENCE
}

Juana Luz Montero Pereda ${ }^{1}$

\section{RESUMEN}

Trabajar derechos humanos y sociales como curso de Responsabilidad Social en el primer ciclo resulta excelente, porque los ingresantes se encuentran en etapa de transición universitaria y aprecian mejor que estos son la vida misma. Son tan la vida misma que aparentemente no se ven, a pesar de estar a flor de piel.

Objetivo: valorar los derechos humanos y defender el derecho a la vida en la violencia familiar con estudiantes de Administración, Administración Turística, Derecho, Obstetricia y Psicología en el año 2013-1.

Medios y materiales: investigación documental cualitativa, tipo descriptiva e interpretativa, utilizando como recurso educativo el cine fórum.

Resultados: Se logró sensibilizar a 800 personas sobre derecho a la vida, en la violencia familiar, con la película "Te doy mis ojos".

Conclusiones: Agradable y emotiva estrategia masiva de aprendizaje, que permite inmediatamente reflexionar, integrar y rescatar el derecho a la vida en la violación de los derechos humanos.

PALABRAS ClAVE: Derecho a la vida, derechos humanos, cine fórum, violación de derechos humanos.

* Recibido: 5 agosto 2014; aprobado: 17 noviembre 2014.

1 Abogada, Mg. en Docencia, Investigación y Currículo, Docente Medio Ambiente, Derechos Humanos y Sociales, Dirección de Responsabilidad Universidad Católica Los Ángeles de Chimbote. 


\section{ABSTRACT}

The importance of considering Human and Social Rights as a subject of Social Responsibility in the first cycle lies in the fact that university entrants are in the stage of transition considering them as life itself even without look at them, they are close to the surface. Aims: To assess the human rights and defending the right to life in family violence with Administration, Tourism Management, Law, Psychology and Obstetrics students in the year 2013-1. Methodology: Qualitative Research documentary, descriptive and interpretive type, using film forum as an educational resource. Results: In total the people sensitized on the right to life, family violence, with the film "Take My Eyes" reached 800 Conclusions: Nice and massive emotional learning strategy, which allows students immediately reflect, integrate and recover the right to life in violation of human rights.

KEY WORDS: Right to life, human rights, film forum, human rights violation.

"El problema de fondo relativo a los derechos humanos no es hoy, tanto el de justificarlos, como el de protegerlos»

Norberto Bobbio

\section{INTRODUCCIÓN}

Los derechos fundamentales son los que poseen los seres humanos por el solo hecho de existir y son anteriores a los derechos humanos. La ONU los define como "garantías esenciales para que podamos vivir como seres humanos. Sin ellos no podemos cultivar ni ejercer plenamente nuestras cualidades, nuestra inteligencia, talento y espiritualidad" (Naciones Unidas Portal de los Derechos Humanos: La ONU y los Derechos Humanos)

La Declaración Universal de Derechos Humanos, en su Artículo $3^{\circ}$, considera "Todo individuo tiene derecho a la vida, a la libertad y a la seguridad de su persona" (Preámbulo Declaración Universal de los Derechos Humanos). La Constitución Política del Perú, en el Capítulo I, Derechos Fundamentales de la Persona, Artículo $1^{\circ}$ declara: "La defensa de la persona humana y el respeto de su dignidad son el fin supremo de la sociedad y del Estado" (Constitución Política del Perú, Capítulo I, Derechos Fundamentales de la Persona).

Los derechos fundamentales y los derechos humanos otorgan dignidad de persona. Su defensa y respeto garantizan el fin supremo de la sociedad peruana; por ello, trabajar la asignatura Derechos Humanos y Sociales como curso de Responsabilidad Social en el primer ciclo es sumamente grato; los ingresantes se encuentran en etapa de transición universitaria y aprecian mejor la vida misma. Son tan la vida misma que aparentemente no se ven, a pesar de estar a flor 
de piel. Y porque no se miran ni se ven se violan frecuentemente, atentando inclusive contra la vida, convirtiéndose en pandemia en el país, afectando especialmente a grupos vulnerables (niños, mujeres, adultos mayores, homosexuales, discapacitados, entre otros).

Para conocer, saber y evitar la violencia familiar, era obligatorio sensibilizar sobre la importancia de los derechos humanos y valorar el derecho a la vida, optando por el cine fórum como recurso didáctico mediante la película "Te doy mis ojos".

\section{MATERIAL Y MÉTODOS}

La investigación estuvo planteada bajo el paradigma cualitativo, utilizando un método de análisis descriptivo e interpretativo. Dentro del enfoque metodológico se propuso este estudio como una investigación básica, aplicada y documental, dado que se buscaba valorar el derecho a la vida en la violencia familiar. El instrumento fue un cuestionario de ingreso a los derechos humanos con cinco preguntas de percepción a 250 estudiantes de las distintas carreras profesionales. El resultado irreflexivo sobre derechos humanos obligaba realizar urgentemente una actividad de proyección social grande, que generara impacto, optando por el cine fórum con la película "Te doy mis ojos", actividad en la que con carácter de imperativo cada estudiante venía acompañado de tres invitados.

Tema: patriarcado, violencia de género, violencia directa.

Valores: autoestima, dignidad, respeto, consecuencias de la violencia, solidaridad.

Conceptos a trabajar: patriarcado, género, violencia de género, estereotipos, empoderamiento.

Actitudes: adquirir una actitud crítica ante las estructuras sociales creadas por el patriarcado que fomentan la violencia de género. Adquirir herramientas de empoderamiento, fomentar la autoestima, fortalecer la personalidad.

Sinopsis. Esta es la historia de un matrimonio, el de Pilar y Antonio, marcada por los malos tratos que sufre ella. Es la historia de un hombre que se intenta imponer siempre y una mujer que se entrega hasta no saber ya quién es. Es una historia de destrucción y autodestrucción, de un círculo del cual es necesario salir para poder sobrevivir, en una sociedad que debe plantearse las estructuras del patriarcado antes que estas acaben con el género femenino. 


\section{RESULTADOS}

1. Conoce de los derechos humanos: el $60 \%$ marcó poco; el $10 \%$, nada y el $30 \%$, mucho. Respuesta alarmante del $70 \%$ entre poco y nada. A pesar que debe haber conocimientos producto de la EBR "Organiza información sobre el patriotismo y los fundamentos de los derechos humanos, discrimina las etapas de la evolución de los derechos humanos" (Formación Cívica y Ciudadana- Primer Grado Educación Secundaria DCN 2009), pág. 403.

2. Conoce la Declaración Universal de los Derechos Humanos: el $80 \%$, poco y el $20 \%$, nada. Impresionante respuesta: $100 \%$ de alumnos no conocen la Declaración Universal de los Derechos Humanos; preocupación máxima se encuentra en "Contenidos generales asignatura Formación Cívica y Ciudadana” (DCN 2009), pág.402.

3. Conoce y valora el derecho a la vida: el $80 \%$ marcó que mucho y el $20 \%$, poco. Respuesta aparentemente satisfactoria. Luego de un coloquio amical concluimos que lo hacen como cuestión de género y supervivencia en función de la etapa de juventud para vivir y divertirse, mas no como relación de vida como derecho humano.

4. Dispone de información sobre la violencia familiar: entre poco y nada $90 \%$. Angustiante respuesta: explícitamente se considera en la capacidad de "Analizar las causas y consecuencias de la violencia familiar valorando los medios de protección familiar y como conocimiento vínculo familiar, ciclo de la vida familiar, crisis y acuerdos familiares" (DCN 2009. Asignatura Persona, Familia y Relaciones Humanas, cuarto grado de educación secundaria, pág. 421).

5. Le importa conocer acerca del derecho a la vida en la violencia familiar: el $100 \%$, entre mucho y poco respondió que sí. Respuesta implícita que solicita auxilio, ya que en la rueda de diálogo de manera advertida o inadvertida esta se encuentra en la mayoría de hogares de los estudiantes, en sus dos formas: violencia física y psíquica.

\section{DISCUSIÓN}

Valorar el pasado, presente y futuro del conocimiento del estudiante universitario acerca del derecho a la vida, derecho de la familia, en derechos humanos, obligaba a averiguar entre docentes la estrategia de enseñanza-aprendizaje utilizada en cuanto a enseñanza de derechos humanos. Respuestas que llevaron 
a las fuentes de la Educación Básica Regular en el Perú, el Diseño Curricular Nacional 2009. Lo encontrado aquí justifica todo: los derechos humanos son temas transversales en la EBR.

\section{TEMAS TRANSVERSALES- IMPORTANCIA Y FINALIDAD}

"Los temas transversales constituyen una respuesta a los problemas actuales de trascendencia que afectan a la sociedad y que demandan a la Educación una atención prioritaria. Tienen como finalidad promover el análisis y reflexión de los problemas sociales, ecológicos o ambientales y de relación personal con la realidad local, regional, nacional y mundial, para que los estudiantes identifiquen las causas; así como los obstáculos que impiden la solución justa de estos problemas. Los temas transversales se plasman fundamentalmente en valores y actitudes. Mediante el desarrollo de valores y actitudes, se espera que los estudiantes reflexionen y elaboren sus propios juicios ante dichos problemas y sean capaces de adoptar frente a ellos, comportamientos basados en valores, racional y libremente asumidos. De esta manera, el trabajo con los temas transversales contribuirá a la formación de personas autónomas, capaces de enjuiciar críticamente la realidad y participar en su mejoramiento y transformación.

Los temas transversales deben ser previstos y desarrollados al interior de todas las áreas curriculares, deben impregnar y orientar la práctica educativa y todas las actividades que se realizan en la institución educativa; por lo tanto, han de estar presentes como lineamientos de orientación para la diversificación y programación curricular. Educación para la convivencia, la paz y la ciudadanía. Educación en y para los derechos humanos. Educación en valores o formación ética. Educación para la gestión de riesgos y la conciencia ambiental. Educación para la equidad de género" (DCN 2009) pág. 35.

Identificar la problemática corroboró que los derechos humanos se enseñan como contenidos de varias disciplinas y su tratamiento es complementario. Realmente es todo y nada en las asignaturas; son temas fugaces, porque la transversalidad inherente no los compromete con derechos fundamentales; mucho menos con derechos humanos, y peor aún, con la Declaración Universal de los Derechos Humanos, como la vida misma que otorga dignidad de ser humano; dignidad que lo enaltece porque solo con ella las personas pueden modelar y mejorar sus vidas mediante la toma de decisiones y sobre todo, el ejercicio de su libertad.

Este proyecto era apremiante; por lo tanto, decidimos trabajar el cinefórum como recurso didáctico óptimo para valorar los derechos humanos y sobre todo, para recuperar el derecho a la vida en la violencia familiar. 
Diligentemente se desarrolló la actividad de proyección social mediante la película "Te doy mis ojos" para generar impacto. Y así fue: primero, un concurso de exposición de testimonios por parte de estudiantes de las distintas carreras profesionales; luego, la película "Te doy mis ojos"; y finalmente, el comentario de expertas, a cargo de la Dra. Teresa García García, Jefa del Departamento de Responsabilidad Social ULADECH Católica, quien analizó la parte psicológica de la mujer; la Dra. Irma Ramírez Castañeda, Juez de Familia de la Corte Superior de Justicia del Santa, encargada de explicar la parte legal en el proceso de violencia familiar; y la abogada Teresa García Morales, Jefa de la DEMUNA de la Municipalidad Provincial del Santa, quien describió casos y explicó el camino fácil para llegar hacia ella. .

Rica, grata y sentida experiencia para todos, especialmente para los estudiantes, quienes aprendieron junto a sus invitados, que los derechos humanos son vida; vida con calidad en la vida misma, y se respetan para garantizar justicia y paz.

Éxito total, no solo por la asistencia registrada en 800 personas, sino por la manera de sensibilizarnos, llegando al extremo de llorar calladamente, aprovechando la tenue luz en la oscuridad durante la proyección de la película.

Se logró sensibilizar y valorar el derecho a la vida, el derecho de la mujer, el derecho de la familia, el derecho del ser humano, el derecho a calidad de vida.

Ayudamos a vivenciar y decir "no" a comportamientos inhumanos, como:

- Celos enfermizos: mujer es quemada con agua hirviendo por su pareja. Jueves, 22 de marzo de 2012 | 9:14 am.

- Mujer atacada a correazos recibió respaldo de pobladores. Martes, 12 de agosto del 2014 | 20:30. Jueves 31 de julio del 2014 | 12:48 m.

- Desfiguran con una botella rota a una joven en Collique. Jueves, 31 de julio del 2014 | 12:48 m.

La educación es el único camino que el Estado tiene para profundizar las políticas de gobierno en prevención, sanción y erradicación de la violencia familiar; pero no como temas transversales que poco o nada hacen por el futuro de la familia.

"La familia: un sistema: orden, totalidad, organización, estructura y finalidad, es un conjunto de personas que conviven bajo el mismo techo, organizadas en roles fijos (padre, madre, hermanos, etc.) con vínculos consanguíneos o no, con un modo de existencia económico y social comunes, con sentimientos afectivos que los unen y aglutinan. 
Naturalmente, pasa por el nacimiento; luego, crecimiento, multiplicación, decadencia y trascendencia. A este proceso se le denomina ciclo vital de vida familiar.

Tiene además una finalidad: generar nuevos individuos a la sociedad" (La Familia un Sistema) Pág. 1.

La finalidad es obvia, justa, urgente y necesaria; por ello proponemos la creación de una asignatura propia en tercero de secundaria con nombre particular: Derecho a la vida en la violencia familiar.

\section{BIBLIOGRAFÍA CONSULTADA}

Constitución Política Del Perú. Capítulo I. Derechos Fundamentales de la Persona. Recuperado de http://www.tc.gob.pe/legconperu/constitucion.html

Preámbulo en DeClaración Universal DE lOS DeREChOS Humanos. Recuperado de http://www.un.org/es/documents/udhr/

FoRMACIÓN CIUDADANA Y CÍviCA - Primer grado - Conocimientos en Diseño Curricular Nacional 2009. Recuperado de http://ebr.minedu.gob.pe/pdfs/den2009final.pdf

III PARTE PRogramación CURRICUlar. Educación Secundaria. Formación Ciudadana y Cívica en Diseño Curricular Nacional 2009 Recuperado de http://ebr.minedu.gob.pe/pdfs/den2009 final.pdf

PERSONA, FAMILIA y RELACIONES hUMANAs. Quinto grado de educación secundaria en Diseño Curricular Nacional 2009. Recuperado de http://ebr.minedu.gob.pe/pdfs/den2009final.pdf

PERSONA, FAMILIA Y RELACiONeS humANAS. Cuarto grado de educación secundaria en Diseño Curricular Nacional 2009. Recuperado de http://ebr.minedu.gob.pe/pdfs/den2009final.pdf

NACIONES UnIDAS. Portal de los Derechos Humanos La ONU y los derechos humanos. Recuperado de www.un.org/es/rights/overview/.

ORGANIZACIÓN DE LOS ESTADOS AMERICANOS. La familia un sistema Instituto Interamericano del Niño. Recuperado de http://www.iin.oea.org/Cursos_a_distancia/Lectura\%2012_UT_1.PDF Pág. 1 